
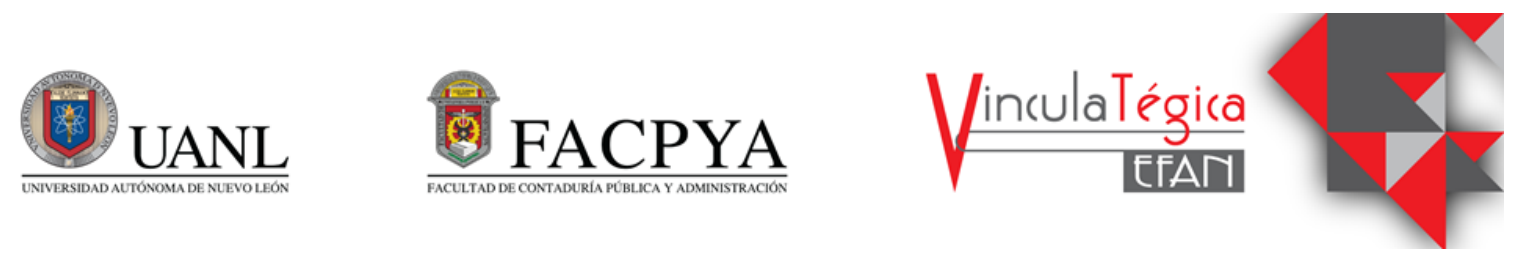

\title{
Marco teórico de los factores que impactan en la internacionalización de las PyMES industriales artesanales de la región citrícola de Nuevo León
}

\author{
Yamileth Aguilar García ${ }^{1}$, Diana Maricela Vásquez Treviño ${ }^{2}$ \\ y Aldahir Caballero Campbell ${ }^{3}$ \\ ${ }^{1}$ Universidad Autónoma de Nuevo León, FACPyA, yamileth.aguilargr@uanl.edu.mx. \\ ${ }^{2}$ Universidad Autónoma de Nuevo León, FACPyA, diana.vasquezdrv@uanl.edu.mx. \\ ${ }^{3}$ Universidad Autónoma de Nuevo León, FACPyA, aldahir.caballerocm@uanl.edu.mx.
}

Información del artículo revisado por pares

Fecha de aceptación: junio-2021

Fecha de publicación en línea: diciembre-2021

DOI: https://doi.org/10.29105/vtga7.2-29

\section{Resumen}

Los productos artesanales alrededor del mundo son expresión de la cultura y tradiciones del lugar donde se fabrican; tienen la característica de ser hechos a mano y en talleres pequeños. La venta de estos productos favorece el turismo. En nuestro país y el mundo, generan empleos en forma de PYMES y existen organizaciones que apoyan y fomentan la fabricación de productos artesanales por medio de regulación y programas de apoyo que promuevan la calidad en los productos para poder internacionalizarse. El Estado de Nuevo León, cercano a la frontera, alberga una región que produce cítricos y tiene una ventaja geográfica. Se propone que la calidad del producto, capacidad de producción, logística y conocimiento del mercado externo inciden en la internacionalización de las PYMES industriales artesanales de la región mencionada.

Palabras claves: PyMES artesanales, Internacionalización, calidad del producto y logística.

\section{Introducción}

El estado de Nuevo León, ubicado al Noreste de México, tiene actividad económica variada que comprende minería, manufactura, servicios, etc. La mayor concentración de habitantes está en el área metropolitana de

\begin{abstract}
Hand made products (handicrafts) around the world are the expression of culture and traditions of the place where they are made; they have the characteristic of being hand -made and manufactured in small workshops. Commercializing these products stimulates tourism. In our country and the resto of the world, these activity creates jobs in SMEs and there are organizations which support and promote the manufacture of handicrafts, through regulations and support programs that encourage product quality to internationalize them. In Nuevo León, state near to the border, there is a region that produces citric fruits and its geographic location is an advantage. It is proposed that quality of the product, productive capacity, logistics and the knowledge of the external market, influence the internationalization of the SMEs that manufacture handicrafts in that region.
\end{abstract}

Keywords: Artisanal SMEs, Internationalization, quality product, logistics.

JEL: F00.

Monterrey sin embargo, la zona sur del estado tiene habitantes y actividad económica que tiene potencial para crecer.

Según datos de INEGI, la población de más de 15 años que tienen ingresos por encima de un salario mínimo se ha 
incrementado de 124,090 en 2005 a 241,788 al finalizar el primer trimestre de 2020. Esto nos indica que tanto la población del estado como las actividades económicas que en él se realizan han tenido un crecimiento importante en los últimos años. Lo que observamos de este dato es que hay muchas personas con disposición y necesidad de laborar y los ingresos que se limitan a ser solo el salario mínimo deberían tener un incremento para mejorar el nivel de vida de los trabajadores.

En datos del Directorio Estadístico Nacional de Unidades Económicas (DENUE), en los municipios que integran la región citrícola del Estado de Nuevo León, que son Allende, Montemorelos, Linares y General Terán, las actividades económicas esenciales son la elaboración de botanas, embutidos, conservas, dulces, panificación, helados, alimentos, así como la confección de ropa, aserrado de tablas y fabricación de utensilios de madera.

Nos parece que al menos las actividades económicas esenciales pueden de cruzar las fronteras de otros países, primero porque la calidad de los productos satisface las necesidades de los turistas que llegan cada año a la región, quienes llevan de regreso a casa estos productos para su consumo o para regalar; segundo porque la ubicación geográfica de la región permite la llegada de los mismos primero a la frontera de con Estados Unidos, pero también el Estado de Nuevo León tiene carreteras adecuadas así como transportistas que podrían beneficiarse de la exportación de los productos que se hacen en la región.

Juntos, el talento de los productores locales y los conocimientos que han adquirido en la región las personas que tienen ya acceso a formación profesional, incrementan las posibilidades de brindarle a la población una oportunidad de comercializar productos fuera del entorno que han venido considerando como convencional y mejorar así la calidad de vida de las familias locales y prolongar la existencia de dichos establecimientos.

\section{Metodología}

Este este trabajo ha recolectado información bibliográfica acerca de las variables presentadas en el mismo; es documental ya que se presenta bibliografía para abordar las variables y es descriptivo explicativo para describir las variables y la relación de entre ellas.

\section{Pregunta central de investigación}

¿Cuáles son los factores que permiten impulsar la internacionalización de las de las PYMES del sector industrial artesanal de la región citrícola?

\section{Hipótesis de la investigación}

Los factores organizacionales que permiten la internacionalización de las PYMES artesanales en la región citrícola del estado de Nuevo León son la capacidad de producción, calidad de los productos, logística y el conocimiento del mercado externo.

\section{Marco teórico}

\section{Antecedentes del problema a estudiar}

Con el hecho de que existen pequeñas y medianas empresas en la región citrícola del Estado de Nuevo León, también podemos asegurar que según datos del INEGI existen sectores específicos en los que las PYMES de la región son buenas y poco se ha hecho para impulsar su crecimiento más allá de las necesidades que pueden satisfacer en el sector turístico.

El interés de investigar los factores organizacionales que permitan impulsar la exportación de los bienes que se manufacturan atiende a que se observan posibilidades tales como la existencia de un mercado fuera de la región, que se sabe que existe gracias al turismo, así como la localización geográfica de la zona.

Así, la motivación central de este trabajo es conocer a través del estudio de las variables, las circunstancias que deben promoverse para que las pequeñas y medianas empresas de la región citrícola del Estado amplíen sus horizontes, dándole paso a una oportunidad nueva de crecimiento tanto a sus organizaciones como al entorno económico en que se desarrollan.

De antemano, sabemos que en su mayoría, los establecimientos que están en 
posibilidad de producir bienes para exportar son aquellos que hacen conservas, dulces, alimentos, utensilios de madera, embutidos y botanas, en su mayoría. Aun así, hay otros productos que buscan los consumidores fuera de la región y que se sabe que son bien aceptados por la gente que visita las ciudades que integran la zona, por lo que consideramos que en general, las PYMES de la región deben ser estudiadas.

\section{Hechos que contextualizan el problema Definición de artesanía}

Las artesanías son productos que tienen diversos usos y que algunas veces tienen implícita la cultura del lugar donde se producen; que pueden ser hechos ya sea totalmente a mano o bien con la ayuda de herramientas.

La UNESCO, que se dedica a establecer la paz mediante la cooperación internacional en materia de educación ciencia y cultura, define artesanía como "Los productos artesanales son los producidos por artesanos, ya sea totalmente a mano, o con la ayuda de herramientas manuales o incluso de medios mecánicos, siempre que la contribución manual directa del artesano siga siendo el componente más importante del producto acabado..." En su portal oficial se reconoce como una organización que tiene una visión global del papel económico, social y cultural de las artesanías en la sociedad, y tiene programas de cooperación entre organizaciones alrededor del mundo en pro de las artesanías. (UNESCO, 2017)

Para Yassir M. Mahgoub de la Universidad de Khartoum, Sudan y Khalid M. Alsoud de la Universidad King Faisal, AlHasa, Arabia Saudita, las artesanías son expresiones únicas de una cultura en particular o una comunidad a través de las habilidades y materiales locales y también reconocen su importancia en el sector productivo y de exportación de los países en desarrollo. (Mahgoub \& M., June 2015)

En general, la literatura revisada nos deja claro que el común denominador de las artesanías alrededor del mundo, es promover la conexión de las culturas y sus tradiciones, que termina siendo un incentivo al turismo sobre todo en las áreas que se encuentran alejadas de las grandes ciudades y en las economías en desarrollo, representa fuentes de empleo y la posibilidad de exportar.

Los productos artesanos, los vamos a definir como todo aquel objeto, sin importar su fin o uso, que ha sido fabricado por una persona que le da forma de manera manual, tiene características únicas y en algunos casos podría ser que una parte del proceso de producción sea industrializado.

En México, en un Acuerdo por el que se emiten las reglas de operación de los programas del Fondo nacional de fomento a las artesanías (FONART) se define artesanía como: "Producto de identidad cultural comunitaria, hecho por procesos manuales continuos, auxiliados por implementos rudimentarios y algunos de función mecánica y utilizando materia prima generalmente nativa." (DOF, 2016)

\section{Importancia del sector industriales artesanal en el mundo}

Los grupos de seres humanos, al rededor del mundo, desde hace mucho tiempo, tienen tradiciones y cultura que los distingue unos de otros. Las manifestaciones artísticas y culturales son variadas y propias de cada región. En el proceso de preservación de la cultura, tradiciones y conocimientos han ido pasando de una generación a otra a lo largo del tiempo. Estas actividades también incluyen la perspectiva económica del desarrollo de la sociedad a lo largo de la historia.

Los bienes artesanales no se producen en masa, aunque puede obtener ayuda de herramientas y algunas máquinas en algunos mercados para hacer negocios; en los países en desarrollo, la comercialización y exportación de piezas hechas a mano es una actividad económica importante. (Mahgoub \& M., June 2015).

Para hablar del escenario del sector artesanal en diferentes partes del mundo, empezaremos por mencionar que en Europa, este sector es esencial para el desarrollo sustentable.

En un análisis de los datos estadísticos y tipología de las empresas, en la siguiente 
tabla se muestra la información de algunas

economías europeas:

\begin{tabular}{|l|c|c|c|c|c|}
\cline { 2 - 6 } \multicolumn{1}{c|}{} & $\mathbf{0}$ & $\mathbf{1 ~ - 9}$ personas & $\mathbf{1 0}-\mathbf{4 9}$ personas & $\mathbf{5 0}-\mathbf{2 4 9}$ personas & Año \\
\hline Bulgaria & & $225,550.00$ & $22,937.00$ & $4,738.00$ & $2,007.00$ \\
\hline Austria & $41,545.00$ & $38,661.00$ & $9,604.00$ & $1,390.00$ & $2,007.00$ \\
\hline Rumania & $558,021.00$ & & $47,603.00$ & $9,850.00$ & $2,007.00$ \\
\hline \multicolumn{1}{|c|}{ Italia } & & $1,158,658.00$ & $317,555.00$ & $\begin{array}{c}\text { No incluido en la definición } \\
\text { de artesanía }\end{array}$ & $2,009.00$ \\
\hline
\end{tabular}

Fuente de elaboración propia. Tamaño de las compañías artesanales por país.

\begin{tabular}{|c|c|c|c|}
\cline { 2 - 4 } \multicolumn{1}{c|}{} & Número total de compañías & Compañías artesanales/ PyMES & $\%$ \\
\hline Bulgaria & & 282,600 & \\
\hline Austria & 294,387 & 92,617 & 31.50 \\
\hline Rumania & - & 615,474 & \\
\hline España & & 65,000 & \\
\hline Italia & $6,065,232$ & $1,480,582$ & 24.40 \\
\hline
\end{tabular}

Fuente de elaboración propia. Porcentaje de las empresas artesanales/PyMES del total de las compañías (IFES, 2010).

Para Latinoamérica, la UNESCO, en su revista Cultura y Desarrollo reconoce lo siguiente: Los países de la región, en mayor o menor medida, reconocen la necesidad de proteger y desarrollar la actividad artesanal a la economía perfeccionando la calidad e incrementado la competitividad nacional e internacional. El hecho de que los gobiernos reconozcan la importancia del sector artesanal en el desarrollo social, económico y cultural hace mejores condiciones para el sector. Lo anterior es importante ya que el sector artesanal aparte se relaciona con el turismo y lo incentiva, representa una actividad económica que genera empleos con una inversión relativamente baja y como la manufactura es manual, hay poca tecnología involucrada. (Benítez Aranda, 2009)

\section{Evolución de la industria artesanal en México}

En México, la producción de artículos artesanales se remonta a la época prehispánica, cuando la producción de artesanías era considera como la creación de artículos personales, objetos utilitarios o de adorno. Con la llegada de la revolución industrial se experimentaron cambios al país, el sector agrícola y artesanal no fueron la prioridad pues se fomentó la creación de ciudades grandes dejando de lado a varios sectores de la población entre e los el indígena. La actividad artesanal sigue vigente, se siguen haciendo productos originados en culturas ancestrales, que contrastan con los producidos por la revolución tecnológica y los avances en diversas áreas del conocimiento (Alemán Tinajero, 2017).

En 1974, el gobierno federal a través de la Secretaría de Desarrollo Social constituyó el fondo Nacional para el Fomento de las Artesanías (Fonart) para promover la actividad de los artesanos del país y así mejorar los ingresos de las familias que dependen de ello, esto a través de cinco vertientes: Capacitación Integral y Asistencia Técnica, Apoyos a la Producción, Adquisición de Artesanías, Apoyos a la Comercialización, y Concursos de Arte Popular. El objetivo es mejorar las fuentes de ingreso favoreciendo sus condiciones productivas y comerciales.

En 1988, se publicó en el Diario Oficial de la Federación la Ley general para el fomento a la microindustria y actividad artesanal, con el objeto fomentar el desarrollo de la microindustria y de la actividad artesanal, aborda mediante el tema fiscal, financiero, mercado y asistencia, así como las facilidades para constituir empresas en forma de personas morales y los trámites correspondientes con autoridades federales y locales. (Cámara de Diputados de H Congreso de la Unión, 2012)

En 2013, en un Acuerdo por el que se emiten las reglas de operación de los 
programas del Fondo nacional de fomento a las artesanías (FONART) se publicaron los criterios de selección para proyectos artesanales que incluye requisitos para obtener apoyos que implican recursos económicos para mejorar la comercialización de los productos de los artesanos. (Diario oficial de la Federación, 2013)

Actualmente en México, los productos hechos a mano son de distintos tipos $\mathrm{y}$ cumplen varias funciones, podemos mencionar la alfarería, muebles, prendas de vestir, zapatos, joyería, juguetes y comida, por mencionar algunos. México y sus artesanías funcionan generalmente igual que en otras partes, pues los lugares en nuestro país en donde hay más atracción de turismo, también tienen sus expresiones artísticas y culturales por medio de diversas piezas realizadas por los habitantes de la localidad y que aparte de tener un propósito útil cumplen con la característica de representar la cultura del lugar.

Según publicación de la revista Forbes en 2014, las artesanías mexicanas están muy bien posicionadas en el mundo y cuentan don reconocimiento por ser una muestra de la diversidad cultural de la sociedad y lo califican como un mercado en crecimiento; según la publicación, países como Australia, Alemania, Canadá, Colombia, Estados Unidos e Italia reciben artesanías hechas en México. (Forbes México, 2014)

Según reporta INEGI en su portal oficial, el valor económico de la cultura y su participación en la economía nacional, en 2019 se reporta una participación del $3.1 \%$ en el PIB con una cifra en millones de pesos de 724,453 y 1,395,644 trabajos ocupados. (Instituto Nacional de Geografía y Estadística, 2019)

En el enfoque que se persigue para esta investigación, tenemos en primer plano el hecho de que las PYMES en general, no solo las de la región en cuestión, están limitadas en el volumen de sus operaciones por lo que podría resultar normal, el hecho de que el mercado al que se dirige generalmente es local. Resulta obvio pensar que si una empresa amplía su mercado, el volumen de sus ventas se incrementará y con ello habrá más flujo de dinero a la misma, sin embargo, no es tan sencillo como solo querer hacerlo si consideramos que cada pequeña y mediana empresa, dependiendo de su giro, ubicación, recursos económicos, etc. enfrentará dificultades que tendrá que sortear para poder ampliarse al mercado internacional.

También cabe mencionar que no es necesario o conveniente para todas las empresas que se haga la lucha por exportar, pero dadas las circunstancias, si la oportunidad se hacer accesible e interesante para alguna, se deben de buscar los obstáculos existentes para darle paso a la oportunidad de comercializar productos en mercados fuera del local, que aparte de incrementar las ventas, también diversifica el riesgo que existe de depender de un solo tipo de mercado y da la oportunidad de sacar ventaja del hecho de que si la economía local va mal, tendrá la ventaja de estar presente con su producto en otro lado que no esté pasando por lo mismo y así diversificar sus ingresos, algo que ayuda a enfrentar las vicisitudes económicas en rachas económicas poco favorables.

En el portal de INEGI, nos definen exportaciones como el total de mercancías, cuyo monto puede ser expresado en términos de cantidad, peso y valor monetario, que salen del territorio nacional de forma definitiva o temporal, mediante un pedimento aduanero, cumpliendo con las disposiciones de la Ley y Normatividad Aduanera vigente. Entonces, entendemos que la principal actividad de debemos relacionar con la posibilidad de una PYME para tener un mercado más amplio es salir del territorio nacional con sus productos.

Por otra parte, Araya Leandro (2009) dice que la internacionalización de empresas consiste en el proceso por el cual una empresa participa de la realidad de la globalización, es decir, la forma en que la empresa proyecta sus actividades, total o parcialmente, a un entorno internacional y genera flujos de diversos tipos (comerciales, financieros y de conocimiento) entre distintos países. Si hilamos esta idea con la exportación, entendemos que la primera lleva a la existencia de la segunda y que la internacionalización debe obedecer a la nueva realidad que viven las empresas que pueden competir en un mercado global, atendiendo a las disposiciones pertinentes para el caso. 
Entonces, por internacionalización entendemos que es la capacidad de una empresa que obtiene al poder vender sus productos o lograr colocar sus mercancías físicamente en otro país que no sea el de origen.

Según Hernández Perales y Hernández Díaz (2013) la internacionalización incluye desde la importación hasta la formación de alianzas estratégicas transfronterizas de diferente naturaleza. A pesar de que se pueden encontrar en la literatura varias teorías que embonan con la idea de la internacionalización de las PYMES, en particular, ellos en su trabajo hablan del modelo incremental de Uppsala, que sugiere que este proceso se debe vivir por etapas con base en la experiencia que se va adquiriendo, los recursos disponibles, empezando por niveles de compromiso así como riesgos moderados y así irlos incrementando.

Coase (1937) centra su pensamiento en los costos y su teoría de internacionalización tiene razón de ser si abriendo nuevos mercados los beneficios derivados son superiores a los costos de aprendizaje y esto conlleva a la expansión exterior.

Según la teoría de la ventaja absoluta de Adam Smith (1723-1790), hace relación a los patrones de producción global en el comercio internacional, dice que a partir de éste, un país será capaz de exportar un bien u otro en función de la productividad de éste.

Para David Ricardo (1772 - 1823), la teoría de la ventaja comparativa se expresa como concepto de productividad en la economía, que dice que, las naciones deberían de especializarse y exportar aquello que mejor saben hacer.

Primero, la capacidad de producción es considerada por Chase, Jacobs y Aquilano como la cantidad de producción que un sistema es capaz de generar en un periodo específico. Por ejemplo, si estamos hablando de una compañía que vende servicios, se expresaría en la cantidad de clientes que se pueden atender cada determinado tiempo, si hablamos de manufactura, se expresaría en el número de productos que se hacen por turno. (Chase Richard B, 2009)
Alemán Tinajero (2017) dice que los artesanos producen en pequeños talleres y que no tienen herramientas para producir y por eso se elevan su tiempo y costos de producción (Alemán Tinajero, 2017). Según Leticia Hernández 2018 de El Financiero, la productividad de las PYMES mexicanas creció de un $34.7 \%$ a $41 \%$ y considera que va lento y menciona que con respecto a los países miembros de la OCDE (Organización de Cooperación y Desarrollo Económico), éstos reportan niveles del 50\% al 60\%. (Hernández, 2018)

En esta investigación, la capacidad de producción de las PYMES de la región citrícola del Estado de Nuevo León, debe estudiarse ya que la internacionalización implica satisfacer la demanda del mercado local y de los nuevos mercados a los que se pretende llegar, por lo tanto la respuesta oportuna a más grandes exigencias estará directamente ligado a la capacidad de producir los bienes que el mercado tanto local como foráneo requieran.

Para la Real Academia Española, calidad es la propiedad o conjunto de propiedades inherentes a algo, que permiten juzgar su valor. Para ISO 9001:2015, calidad es el grado en el que un conjunto de características inherentes cumple con los requisitos.

Gutiérrez Pulido, menciona que en 1990 Jurán definió la calidad como la ausencia de deficiencias en aquellas características que satisfacen al cliente. (Gutiérrez Pulido, 2010)

Además, en el programa de apoyo a la actividad artesanal, desde el periodo de 1991 a 1994, se muestra un apartado se promueve la calidad total. También, mencionamos que el Diario Oficial de la Federación publicó la NOM 051 en 2010, con las especificaciones para el envasado y etiquetado de bebidas no alcohólicas y alimentos, en donde se reglamenta que se debe considerar en etiquetado las designaciones de calidad de manera comprensible y sin engaños; este etiquetado es necesario para la exportación de los productos. (Diario Oficial de la Federación, 2010).

Tamanini en 2009 menciona que la calidad tiene un efecto positivo en la 
satisfacción del cliente y permite incrementar la participación en el mercado; aseguran que un sistema de gestión de calidad facilita que las PYMES compitan con empresas grandes. (Tamanini, 2009). También, Martínez Duclaud 2015, menciona en su publicación la importancia que tienen las certificaciones de calidad para las PYMES, dice que mejoran la productividad de las mismas y que implica planeación antes, durante y después de obtenerla. (Martínez Dulcaud, 2015)

En esta investigación, considerando que los artículos que salen del país deben cumplir con ciertos estándares, dependiendo de cuál sea su destino, es importante considerar la calidad de los productos artesanales como uno de los factores que inciden en el proceso de internacionalización de los mismos.

La logística, según la real academia española, es un conjunto de medios y métodos necesarios para llevar a cabo la organización de una empresa o servicio, especialmente la distribución. Rodríguez, Cruz y Lam, mencionan que la logística se relaciona con procedimientos que abarcan desde la compra de materias primas hasta la entrega del producto final al consumidor (Rodríguez Sáenz, Cruz, \& Lam, 2009).

Avendaño y Silva en 2018 sugieren que la logística es un proceso para llevar a cabo una exportación siguiendo una serie de actividades que van desde la documentación hasta aspectos técnicos y legales que exige el país importador teniendo en cuenta la exigencia de los clientes (Avendaño Cárdenas \& Silva Guerra, 2018)

Gil Gaytán 2017, menciona a Luraschi 2005 y dice que algunos de los aspectos que evalúan los países latinoamericanos son que no todos los países de la región han logrado avanzar en la logística a niveles avanzados, la cultura de las empresas carece de una visión integral de la cadena de suministro, el transporte y almacenamiento no son muy efectivos, entre otros. (Gil Gaytán, 2017). Dice Kirby y Brosa 2011 que la eficiencia logística de una región o país se condiciona por qué tan desarrolladas están sus infraestructuras, sus leyes y reglamentos, así como la calidad de sus servicios logísticos y según sus estudios,
América Latina tiene una mejor valuación que otras regiones emergentes. (Kirby \& Brosa, 2011). Sieso 2015 sugiere que para una buena gestión logística, es necesario identificar los costos y estos se ven afectados por variables como la ubicación, entre otras. (Sieso, 2015)

Para esta investigación, consideramos importante el estudio de la logística que deben de seguir los productos artesanales de la región citrícola, porque es esto lo que precisamente hace posible el intercambio comercial entre países.

El mercado externo, para Westreicher (2020) representa las actividades económicas de un país con el resto del mundo, incluidos la compra venta de bienes y servicios. (Westreicher, 2020). También, el mercado externo es el conjunto de transacciones comerciales internacionales que incluye el total de las importaciones y exportaciones de bienes, servicios y capital, que se realizan. (Diccionario Jurídico y Social, 2017).

Hernández Perales y Hernández Díaz (2013) mencionan que las empresas mexicanas tienen un intercambio comercial fuerte con Estados Unidos, pero es necesario conocer la percepción de los directivos acerca del mercado europeo, por ejemplo. (Hernández Perales \& Hernández Díaz, 2013). Según Elizabeth Meza, el comercio exterior de México con Europa ascendió a 80,000 millones de dólares al cierre de 2018, habla de la diversificación de las relaciones comerciales de nuestro país, no solo centrarse en Estados Unidos. (Meza, 2020). Mariana Ramos, dice que de acuerdo a la Asociación Mexicana de Venta Online, el 54\% de los negocios locales están planeando ampliarse por medio de la exportación, por medio de las ventas en línea; dice que las PyMES mexicanas también pueden pensar en otros destinos para exportar y señala que las que producen bebidas, textiles y alimentos, entre otros ramos, son las que tienen que atender a más regulaciones de los lugares en donde se va a recibir su producto (Ramos, 2020).

Este trabajo busca los factores que inciden en la internacionalización de las PYMES artesanales de la región citrícola, por lo tanto conocer la interacción económica con 
otros países es considerado importante para la investigación.

\section{Conclusiones}

Derivado del análisis del marco teórico identificamos los principales factores de la internacionalización de las PYMES industriales artesanales de la región citrícola del Estado de Nuevo León, y según los más destacados autores en la literatura analizada, las principales variables son: capacidad de producción, calidad en el producto, logística y conocimiento del mercado externo.

- El sector industrial artesanal tiene relevancia en el mudo debido a que los bienes que producen y venden tienen su origen en las tradiciones del sector de la población que los fabrica.

- El sector industrial artesanal genera empleos para las familias que los producen

- El turismo promueve el consumo de los productos artesanales.

- En México, el sector artesanal está en crecimiento, algunos países alrededor del mundo reciben artesanías mexicanas.

- En México se producen artesanías que atraen al turismo y existe regulación para apoyar a dicho sector de diversas formas, que ayudan a su proceso de internacionalización.

- Las PYMES deben poner atención a los aspectos relacionados con la calidad de sus productos ya que esto les puede permitir más fácil el acceso a los mercados extranjeros.

- El proceso de logística es costoso, por lo tanto las PYMES deben aprender acerca de cómo funciona.

- Actualmente, las exportaciones están centradas en Estados Unidos, pero es recomendable buscar oportunidad en otros lugares del mundo.

Las variables calidad en el producto, capacidad de producción, logística y conocimiento del mercado externo impactan positivamente a la internacionalización de las PYMES industriales artesanales del Estado de Nuevo León.

Para las futuras investigaciones se hará un instrumento de medición, una prueba piloto y la recolección de datos para confirmar los factores que influyen de internacionalización de las PYMES analizadas en la literatura. 


\section{Referencias}

Aleman Tinajero, C. J. (Junio de 2017). Desarrollo de un plan de negocios para la exportación de artesanías de cobre al mercado alemán. Tesis Maestría en Mercadotecnia Global. Instituto Tecnilógico de Estudios Superiores de Occidente, Tlaquepaque, Jalisco, México.

Araya Leandro, A. (2009). El Proceso de internacionalización de empresas. TEC Empresarial, 18 - 25

Avendaño Cárdenas, E., \& Silva Guerra, H. (2018). Análisis de lso cuellos de botella en la logística internacional de las Pymes de confecciones en Colombia. Revista de estudios interdiciplicarios en Ciencias Sociales, 510-536.

Benítez Aranda, S. (2009). La Artesanía latinoamericana como factor de desarrollo económico, social y cultural: a la luz de los nuevos conceptos de cultura y desarrollo. Revista Cultura y Desarrollo, 18. Extraido 5 de Marzo de 2021desde http://www.lacult.unesco.org/docc/CyD_6.pdf.

Camara de Diputados de H Congreso de la Unión. (09 de Abril de 2012). Cámara de diputados. Obtenido de Cámara de diputados: http://www.diputados.gob.mx/LeyesBiblio/pdf/127.pdf

Chase Richard B, J. R. (2009). Administración de operaciones. México: Mc. Graw Hill.

Diario del exportador. (Agosto de 2017). Diario del exportador. Obtenido de Diario del exportador: https://www.diariodelexportador.com/2017/08/contenido-de-un-estudiode-mercado-para.html

Diario Oficial de la Federación. (01 de Junio de 1992). Diario Oficial de la Federación. Obtenido de Diario Oficial de la Federación: http://dof.gob.mx/nota_detalle.php?codigo=4669191\&fecha=01/06/1992

Diario Oficial de la Federación. (21 de Diciembre de 2006). Diario Oficial de la Federación. Obtenido de Diario Oficial de la Federación: http://www.diputados.gob.mx/LeyesBiblio/pdf/28.pdf

Diario oficial de la Federación. (2013). Diario Oficial de la Federación. Obtenido de Diario Oficial de la Federación: https://www.dof.gob.mx/nota_detalle_popup.php?codigo=5288943

Diccionario Jurídico y Social. (Junio de 2017). Obtenido de Diccionario Jurídico y Social: https://diccionario.leyderecho.org/mercado-externo/

DOF. (27 de Diciembre de 2016). DOF. Obtenido de dof.gob.mx: https://www.dof.gob.mx/nota_detalle.php?codigo=5467716\&fecha=27/12/2016

Forbes México. (2014). México y sus artesanías en el mundo. Forbes. Extraído 5 de Marzo 2021 desde https://www.forbes.com.mx/mexico-y-sus-artesanias-en-el-mundo/.

Gil Gaytán, O. L. (2017). La logística: clave para la competitividad global de las pequeñas y medianas empresas del estado de Jalisco en México. PAAKAT: revista de tecnología y sociedad (Vol. 6) Extraído el 7 deMayo de 2021 desde http://www.scielo.org.mx/scielo.php?script=sci_arttext\&pid=S2007$36072017000100004 \#$ B1.

Gutiérrez Pulido, H. (2010). Calidad total y productividad. México: Mc Graw Hill.

Hernández Perales, N. A., \& Hernández Díaz, R. (2013). Internacionalización de la PYME en México, un estudio exploratorio. XVIII Congreso Internacional de Contaduría, Administración e Informática. Extraído 5 de Marzo 2021 desde http://congreso.investiga.fca.unam.mx/docs/xviii/docs/1.16.pdf. 
Hernández Sampieri, R. (2014). Metodología de la Investigación. México D.F.: MC Graw Hill.

Hernández, L. (20 de Febrero de 2018). El Financiero. Obtenido el 12 de Mayo de 2021 de elfinanciero.com.mx: elfinanciero.com.mx/economia/productividad-de-las-pymes-enmexico-va-lento

IFES. (Marzo - Abril de 2010). Report on the situation of craftmanship in Europe. 8-9. Obtenido de http://projects.ifes.es/: http://projects.ifes.es/pdfs/craft/craft1.pdf

Instituto Nacional de Geografía y Estadística. (2019). INEGI. Obtenido de INEGI: https://www.inegi.org.mx/temas/cultura/

Kirby, C., \& Brosa, N. (2011). La logística como factor de la competitividad de las PYMES en las Américas. V Foro de Competitividad de las Américas para el Banco Interamericano de Desarrollo (págs. 1- 39). Santo Domingo, República Dominicana: BID.

Mahgoub, Y. M., \& M., A. K. (June 2015). he Impact of Handicrafts on the Promotion of Cultural and Economic Development for Students of Art Education in Higher Education . Journal of Literature and Art Studies, Vol. 5, No. 6, 471-479.

Martínez Dulcaud, R. (2015 de Febrero de 2015). El empresario. Obtenido el 18 de Mayo 2021 de elempresario.mx: https://elempresario.mx/certificacion/pymes-importanciasu-certificacion

Meza, E. (18 de Febrero de 2020). El Emprsario. https://elempresario.mx/nuevosmercados/europa-busca-pymes-mexicanas

Misión de México ante la UE. (Septiembre 2010). 200 años de comercio exterior de México. Lazos Comerciales, $\quad 1-4 \quad$ (http://www.economiasnci.gob.mx/sic_php/pages/bruselas/trade_links/esp/sepesp2010.pdf).

Ramos, M. (14 de Julio de 2020). Marketing4ecommerce. Obtenido el 18 de Mayo 2021 de marketing4ecommerce.mx: https://marketing4ecommerce.mx/exportar-el-principalobjetivo-de-la-mayoria-de-pymes-mexicanas/

Rodríguez Sáenz, D., Cruz, C., \& Lam, F. (2009). Logística para la expostación de productos agrícolas, frescos y procesados. San José, Costa Rica: Instituto Interamericano de Cooperación para la Agricultura IICA.

Sieso, M. (14 de octubre de 2015). Por la empresa. Obtenido el 18 de Mayo de 2021 de porlaempresa.com: https://porlaempresa.com/la-gestion-logistica-como-ventajacompetitiva/

Tamanini, H. F. (2009). Lo mejor de la calidad en PYMES. Petrotecnia, 40 - 45.

Trade Facilitation Office. (s.f.). Trade Facilitation Office. Obtenido el 18 de Febrero de Trade Facilitation Office: https://www.tfocanada.ca/docs.php?page=2_8

UNESCO. (2017). unesco.org. Obtenido el 18 de Febrero de 2021 de unesco.org: http://www.unesco.org/new/es/culture/themes/creativity/creative-industries/craftsand-design/

Westreicher, G. (16 de Agosto de 2020). Economipedia. https://economipedia.com/definiciones/mercado-externo.html 